\title{
Rheumatoide Arthritis: kardiovaskuläres Risiko bei KHK besonders hoch
}

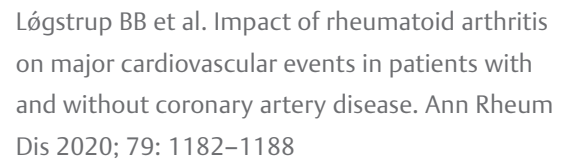

Rheumatoide Arthritis (RA) ist ein Risikofaktor für kardiovaskuläre Erkrankungen. Wie sich das Risiko für kardiovaskuläre Ereignisse erhöht, wenn RAPatienten zusätzlich an einer koronaren Herzerkrankung (KHK) leiden, ist nicht bekannt. Eine dänische Studie hat den Zusammenhang untersucht.

In der Studie wurden Daten von volljährigen Patienten aus einem dänischem Herzregister (Western Denmark Heart registry) ausgewertet, die sich zwischen 2003 und 2016 einer Koronarangiografie (KAG) unterzogen hatten und danach nachbeobachtet wurden. Die Patienten wurden in der Auswertung nach einem Vorliegen von RA und KHK stratifiziert. Patienten, bei denen vor der KAG schon Interventionen an den Koronararterien stattgefunden hatten (z. B. Bypass) oder die einen Myokardinfarkt (MI) erlitten hatten, wurden ausgeschlossen. Zu den Patienten lagen auch Informationen zu Begleiterkrankungen wie Bluthochdruck, Schlaganfällen, TIA, PAVK, Vorhofflimmern, Herzinsuffizienz, Diabetes mellitus, sowie zu ihrer Medikamenteneinnahme vor. 
Insgesamt wurden 125331 Patienten in die Studie eingeschlossen. Endpunkte waren das Auftreten eines MI, anderer unerwünschter kardiovaskulärer Ereignisse (MI, ischämischer Schlaganfall und Herztod) und die Gesamtmortalität.

Die Auswertung zeigte, dass 1061 Patienten $(0,85 \%)$ aus der Population an RA und einer KHK gleichzeitig litten, 671 an RA alleine (0,54\%) und $75082(59,9 \%)$ an einer KHK. Patienten mit RA waren häufiger weiblich und litten häufiger an Herzinsuffizienz, Bluthochdruck, ischämischen Schlaganfällen und Vorhofflimmern als Patienten ohne RA.

Während des Follow-Ups erlitten 6907 Patienten einen MI, 3841 einen ischämischen Schlaganfall, 26692 starben, bei 4937 lag eine kardiale Ursache vor.

Bei Patienten, die weder an RA, noch an einer KHK litten, lag das 10-Jahres-Risiko für einen MI bei 2,7\%. Bei Patienten, die nur an einer RA litten, bei 3,8\% (IRRadj 1,63, $95 \%$ KI 1,04 bis 2,54), bei Patienten mit KHK allein bei 9,9\% (IRRadj 3,35, $95 \% \mathrm{KI} 3,10$ bis 3,62). Bei Patienten, die sowohl an einer RA, als auch an einer KHK litten, lag es bei 12,2\% (IRRadj 4,53, 95 \% KI 3,66 bis 5,59). Ähnliche Zusammenhänge wurden für andere kardiovaskuläre Ereignisse am Herzen, nicht aber für Schlaganfälle gefunden.

FAZIT

Patienten mit RA haben im Vergleich zu Patienten ohne RA ein leicht erhöhtes Risiko für schwere kardiovaskuläre Ereignisse wie einen Myokardinfarkt. Das kardiovaskuläre Risiko der RA-Patienten steigt dramatisch an - so zeigt die vorliegende Studie - wenn die Patienten zusätzlich an einer KHK leiden. Die Autoren schlagen aufgrund des hohen additiven Risikos vor, RA-Patienten gezielt auf das Vorliegen einer KHK zu untersuchen. 